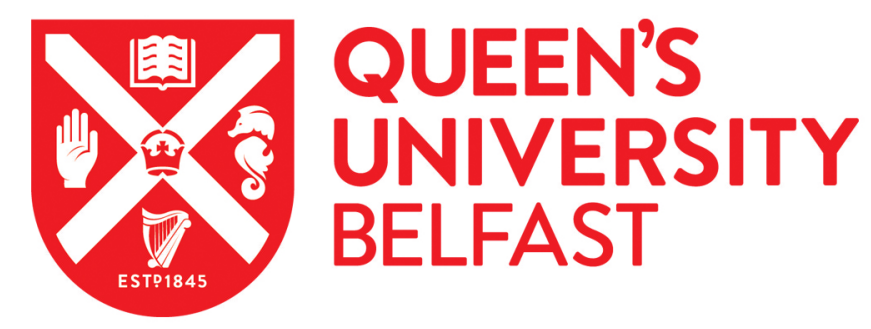

\title{
Extreme-Ultraviolet-Initated High-Order Harmonic Generation: Driving Inner-Valence Electrons Using Below-Threshold-Energy Extreme- Ultraviolet Light
}

Brown, A. C., \& Van Der Hart, H. W. (2016). Extreme-Ultraviolet-Initated High-Order Harmonic Generation: Driving Inner-Valence Electrons Using Below-Threshold-Energy Extreme-Ultraviolet Light. Physical Review Letters, 117(9), [093201]. https://doi.org/10.1103/PhysRevLett.117.093201

Published in:

Physical Review Letters

Document Version:

Peer reviewed version

Queen's University Belfast - Research Portal:

Link to publication record in Queen's University Belfast Research Portal

Publisher rights

(2016 American Physical Society. All rights reserved.

\section{General rights}

Copyright for the publications made accessible via the Queen's University Belfast Research Portal is retained by the author(s) and / or other copyright owners and it is a condition of accessing these publications that users recognise and abide by the legal requirements associated with these rights.

Take down policy

The Research Portal is Queen's institutional repository that provides access to Queen's research output. Every effort has been made to ensure that content in the Research Portal does not infringe any person's rights, or applicable UK laws. If you discover content in the

Research Portal that you believe breaches copyright or violates any law, please contact openaccess@qub.ac.uk. 


\title{
XUV-initiated high harmonic generation: driving inner valence electrons using below-threshold-energy XUV light
}

\author{
A. C. Brown and H. W. van der Hart* \\ Centre for Theoretical Atomic, Molecular and Optical Physics, School of Mathematics and Physics, \\ Queen's University Belfast, Belfast BTry 1NN, United Kingdom
}

(Dated: August 2, 2016)

\begin{abstract}
We propose a novel scheme for resolving the contribution of inner- and outer-valence electrons in XUV-initiated high-harmonic generation in neon. By probing the atom with a low energy (below the $2 s$ ionisation threshold) ultrashort XUV pulse, the $2 p$ electron is steered away from the core, while the $2 s$ electron is enabled to describe recollision trajectories. By selectively suppressing the $2 p$ recollision trajectories we can resolve the contribution of the $2 s$ electron to the high-harmonic spectrum. We apply the classical trajectory model to account for the contribution of the $2 s$ electron, which allows for an intuitive understanding of the process.
\end{abstract}

PACS numbers: 32.80.Rm, 31.15.A-, 42.65.Ky

High harmonic generation (HHG) is now a well established tool both for the generation of high energy and attosecond laser pulses [1, 2], and as a measurement technique for atomic and molecular structure and ultrafast electron dynamics [3-5]. The well-known 'three-step' model captures well the gross dynamics of the process: an electron is 1) ionized then 2) driven by a strong laser field before 3 ) recolliding with its parent ion, and releasing its energy in the form of a high-harmonic photon [6].

For some time now, researchers have been concerned with both the optimization of the process [7-9], and its application to measurement in so called high-harmonic spectroscopy $[3-5,10,11]$. Both of these considerations raise the same conclusion: the initial step of the threestep model (tunnel ionization) limits both the conversion efficiency and the ability of HHG to probe general electron dynamics, restricted as it is to the emission of the outermost valence electron. Hence, it has been proposed that by subjecting the target to both the strong driving field and a short XUV pulse, the HHG process can be initiated by photoionization rather than tunnel ionization [8]. This has the advantage of being significantly more efficient, and of opening the possibility of driving more deeply bound electrons.

Several theoretical studies have explored the efficiency question by utilizing simple model calculations to describe the three-step process $[12,13]$. A related schemewherein an XUV pulse excites a core electron to a valence hole during the valence electron's transit- has also been studied [14-16]. More recently, an XUV-initated HHG (XIHHG- i.e. the core, rather than the valence, electron is emitted in the initial step) scheme was used to elucidate core-hole dynamics in small molecules [17].

The attraction of studying core-hole dynamics is twofold. Firstly, the HHG cutoff is extended in proportion to the electron binding energy, and thus by driving HHG with a deeply bound electron substantial extension of the HHG plateau can be achieved. Not only does this allow the generation of ever shorter, attosecond pulses, an ex- tended cutoff also allows greater scope for analysis of the spectrum. Secondly, high energy photoionization favors ionization of core- rather than valence-electrons, whereas the outer electrons are more susceptible to tunnel ionization and photoionization by lower energy XUV light. Thus, to a reasonable approximation, only the core electron responds to the XUV photon, and the response of the outer electrons can be neglected.

However, one can envisage dynamics involving the correlated motion of inner- and outer-valence electrons which may be probed with XIHHG. For instance, the window resonances in the photoionization spectrum of argon are due to the interference of $3 s$ and $3 p$ electrons. We have previously shown that these interferences also impact on low energy harmonic generation in shortwavelength $(390 \mathrm{~nm})$ fields [18]. To investigate their role in high harmonic generation, driving wavelengths in the near- to mid-IR range should be employed, but at such low photon energies the response of the inner-valence electron is negligible. Thus a combination of XUV and IR light might be used to probe such interference dynamics.

There are only a handful of computational methods capable of describing the multielectron dynamics necessary for a thorough exploration of HHG with inner-valence electrons $[19,20]$. Among them, the R-matrix with timedependence (RMT) technique has demonstrated significant promise for the description of ultrafast processes in general multielectron systems [21]. RMT has been applied variously to HHG in the computationally challenging near-IR wavelength regime [22], electron rescattering in negative ions [23], IR-assisted ultrafast ionization of positive ions [24] and attosecond transient absorption spectroscopy of doubly- and core-excited states [25].

In the present manuscript we explore the XIHHG process in neon and assess the contribution of the innervalence electron to the resulting spectra. In contrast with previous studies, which promote the inner-valence electron during the transit of the outer valence electron [1416], we drive the inner-valence electron on a three-step- 
like trajectory by employing XUV pulse-energies below the $2 s$ ionization threshold. Additionally, we consider how the timing of the ultrashort XUV pulse can be used to selectively enhance the contribution of the $2 s$ electron, an extension of the method previously explored by Schafer et al. [26].

The RMT method is an ab-initio technique for solving the time-dependent Schrödinger equation for general, multielectron atoms or ions in strong laser fields. We employ the well-known R-matrix paradigm, whereby the interaction space is split into two regions: an inner regionclose to the nucleus, wherein full account is taken of all multielectron interactions- and an outer region- where a single, ionized electron moves under the influence of the laser field and the long-range potential of the core. Several implementations of time-dependent R-matrix theory exist [27-30], but RMT offers the most robust and general numerical approach for tackling processes in strong-fields. This is achieved through representing the wavefunction with a B-spline based, R-matrix basis in the inner region, and with a highly efficient grid based approach in the outer region [21].

The neon target used for this study is discussed in detail elsewhere [31]. Briefly: the neon atom is described in a close-coupling with pseudostates scheme as a $\mathrm{Ne}^{+}$ion plus an electron. The descriptions include all $2 s^{2} 2 p^{5} \epsilon \ell$ and $2 s 2 p^{6} \epsilon \ell$ channels up to $L_{\max }=139$. The inner (outer) region boundary is 32 a.u. (2000 a.u.), (results are converged with respect to these boundaries). The set of continuum orbitals contains 70 B-splines for each angular momentum of the continuum electron.

The primary laser pulse used is a 3 -cycle (18 fs), $1.8 \mu \mathrm{m}$ pulse with an intensity of $1.2 \times 10^{14} \mathrm{Wcm}^{-2}$. The XUV intensity is $10 \%$ of the IR intensity. Both the IR and XUV pulses have a $\sin ^{2}$ ramp on/off profile. Unless otherwise stated, the delay between IR and XUV pulses is chosen to be 2.75 fs such that the XUV peak arrives one half-cycle before the IR peak. According to the classical three-step model, electrons 'born' into the field at this time describe the optimal trajectories for HHG.

Calculations performed for various XUV intensities ( $1 \%$ and $0.1 \%$ of the IR intensity), show that the overall yield scales linearly with the XUV intensity. Calculations performed with $800 \mathrm{~nm}$ IR pulses instead of $1.8 \mu \mathrm{m}$ show that the mechanisms discussed below also apply at the lower wavelength. We here display only the results for the $1.8 \mu \mathrm{m}$ IR and $10 \%$ intensity XUV as they support longer plateaus and clearer observation of the key features. The probability to tunnel ionize from the $2 p$ shell in the IR field is $0.00003 \%$, and to photoionize with the $45 \mathrm{eV}$ XUV from the $2 p$ or $2 s$ is $0.3 \%$ and $0.002 \%$ respectively.

We perform calculations for XUV-photon energies ranging from beneath the $2 p$ binding energy $\left(E_{2 p}=\right.$ $21.6 \mathrm{eV})$ to above the $2 s$ binding energy $\left(E_{2 s}=48.5 \mathrm{eV}\right)$, and use two XUV pulse durations: 1 fs ('long') and 250 as

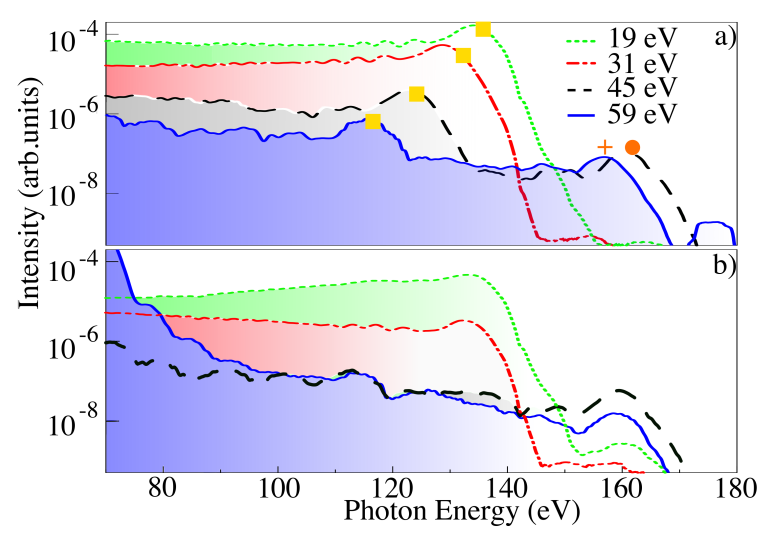

FIG. 1: (Color online) The smoothed harmonic yield from Ne driven by an $18 \mathrm{fs}, 1.8 \mu \mathrm{m}, 1.2 \times 10^{14} \mathrm{Wcm}^{-2}$ pulse, in combination with a a) 1 fs and b) 250 as XUV pulse of energy $19 \mathrm{eV}$ (black dotted line), $31 \mathrm{eV}$ (red dot-dashed line), $45 \mathrm{eV}$ (green dashed line), and $59 \mathrm{eV}$ (blue solid line). The cutoff of the $2 p$ harmonics as predicted by the model (see text) are marked by yellow squares. The predicted $2 s$ cutoff is marked for the $45 \mathrm{eV}$ (circle) and $59 \mathrm{eV}$ ('+') XUV pulses.

('short'). Figure 1 shows the harmonic spectra for four XUV-photon energies- 19, 31, 45, $59 \mathrm{eV}$ (corresponding to particular odd harmonics of the $1.8 \mu \mathrm{m}$ pulse) in both the long- and short-pulse configurations.

At a photon energy of $19 \mathrm{eV}(45 \mathrm{eV})$, the XUV pulse is not sufficiently energetic to ionize the $2 p(2 s)$ electron directly. However, the HHG yield is still increased by several orders of magnitude over the IR-only spectrum (not shown). We attribute this to a two-photon $(\mathrm{IR}+\mathrm{XUV})$ ionization process. These XUV photon energies are resonant with a number of Rydberg states converging onto the $2 p$ and $2 s$ ionization thresholds: and thus photo-excitation to a (superposition of several) Rydberg state(s) is followed by field ionization. This two stage process allows the electron to be 'born' into the field with approximately zero energy, which is crucial for optimizing the three-step recollision process.

For XUV photon energies of $45 \mathrm{eV}$ and $59 \mathrm{eV}$ Fig. 1 shows an extension of the plateau, the cutoff of which is dependent on the XUV photon energy. In the longpulse case there appears a double plateau structure and the cutoff energy of the first plateau (marked by yellow squares) decreases with increasing photon energy.

To understand the extension of the cutoff we consider the empirical formula for the cutoff energy [32]:

$$
E_{\text {cutoff }, 2 \mathrm{p}} \approx 3.2 U_{p}+I_{p}=137 \mathrm{eV},
$$

where $U_{p}$ is the ponderomotive energy, and $I_{p}$ the ionization potential: i.e. the binding energy of the $2 p$ electron: $21.6 \mathrm{eV}$. If instead we use the binding energy of the $2 \mathrm{~s}$ electron then we predict a cutoff of

$$
E_{\text {cutoff }, 2 \mathrm{~s}} \approx 3.2 U_{p}+E_{2 s}=160 \mathrm{eV},
$$

in line with the $45 \mathrm{eV}$ XUV spectrum in Fig. 1. 


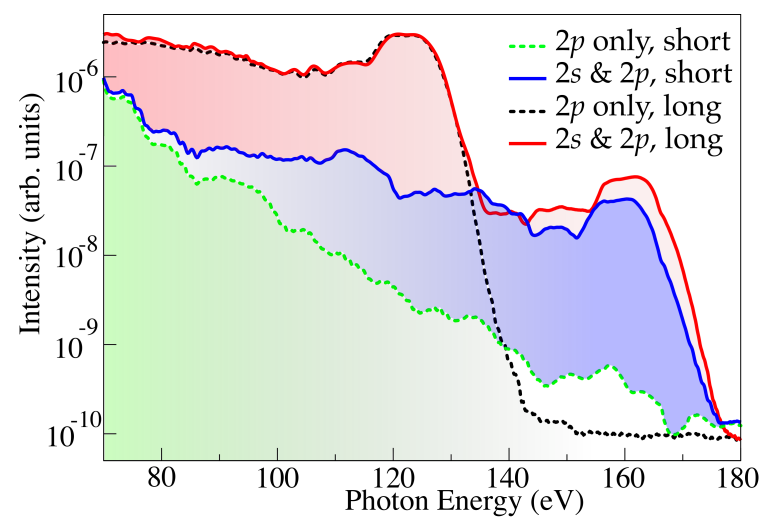

FIG. 2: (Color online) The smoothed cutoff harmonics from Ne driven by an $18 \mathrm{fs}, 1.8 \mu \mathrm{m}, 1.2 \times 10^{14} \mathrm{Wcm}^{-2}$ pulse, in combination with a 250 as (lower two lines), and 1 fs (upper two lines), $45 \mathrm{eV}$ XUV pulse timed to coincide with the penultimate maximum of the IR field. Calculations include the action of both the $2 s$ and $2 p$ electrons (solid lines) or neglecting the action of the $2 s$ (dotted lines).

In order to isolate the contribution of the $2 s$ electron two calculations are performed with an XUV photon energy of $45 \mathrm{eV}$ : one comprising both the $2 s^{2} 2 p^{5}$ and $2 s 2 p^{6}$ $\mathrm{Ne}^{+}$thresholds, and one with only the $2 s^{2} 2 p^{5}$. Removing the $2 s 2 p^{6}$ threshold effectively neglects ionization of the $2 s$ electron in the calculation. A similar approach was used to elucidate the contribution of the $3 s$ and $3 p$ electrons to low energy harmonic generation in $\operatorname{Ar}$ [18].

Figure 2 shows the high energy harmonics from $\mathrm{Ne}$ as generated with these two atomic structure configurations, for both the long and short XUV pulses. There is a clear difference between the spectra from the $2 p$-only and $2 s-2 p$ calculations: the $2 s-2 p$ yield shows a recognisable plateau extending to a cutoff at $160 \mathrm{eV}$ which is not present in the $2 p$-only calculations for either long or short pulses. This confirms that the highest energy harmonics are generated by the action of the $2 s$ electron.

In the long pulse, there is no difference between the $2 p$-only and $2 s-2 p$ spectra up to an initial cutoff at $125 \mathrm{eV}$. For the short pulse, the overall harmonic yield is largely suppressed, but the integrated yield above $100 \mathrm{eV}$ is increased by an order of magnitude by the inclusion of the $2 s$ electron. This is surprising as the ionization probability for a $2 s$ electron is around $1 \%$ of that of a $2 p$ electron. Furthermore, the cutoff ascribed to the $2 p$ electron- $125 \mathrm{eV}$ - does not agree with the classically predicted cutoff energy of $137 \mathrm{eV}$.

To elucidate these differences we perform classical trajectory simulations: for each 'birth time', we can calculate the electron's velocity and position by integrating over the acceleration in the IR field. Those trajectories which pass again through the origin describe recolliding electrons from which the recollision energy- and hence the energy of the HHG radiation- can be determined. Importantly, in general, a photoionized electron will be

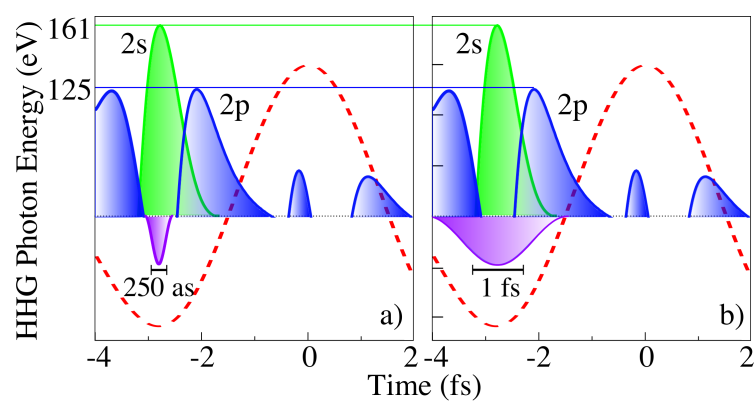

FIG. 3: (Color online) The maximum emitted harmonic photon energies that would result from electrons being released at the times shown along the horizontal axis for both $2 p$ (blue) and $2 s$ (green) electrons from Ne in a $18 \mathrm{fs}, 1.8 \mu \mathrm{m}$, $1.2 \times 10^{14} \mathrm{Wcm}^{-2}$ pulse (red dashed line). Trajectories are initiated by a a) 250 as and b) 1 fs 45 eV XUV pulse. Trajectories of $2 s$ and $2 p$ which lead to recollision are localized in time, and therefore can be resolved in the short-pulse case (a). For a long XUV pulse (b), both electrons contribute.

born into the field with non-zero velocity, while in the traditional three-step model the initial velocity of the tunnel-ionized electron is assumed to be zero [26]. This initial velocity is calculated directly from the excess energy absorbed by the electron: i.e. the difference between the XUV-photon energy and the ionization potential. We note that this velocity may be in any direction relative to the linearly polarized IR field. Thus a $2 p$ electron will gain an excess energy of $23.4 \mathrm{eV}(45-21.6 \mathrm{eV})$, while a $2 s$ electron is born with zero initial velocity (in practice there will be a spread of initial energies due to the bandwidth of the XUV pulse, but we assume just one initial velocity for the purposes of the model).

Figure 3 shows the HHG photon energy for $2 s$ and $2 p$ electron recollision trajectories originating at different times in the IR pulse. The excess energy gained negates the recollision of $2 p$ electrons born at the penultimate peak of the IR, but allows those born earlier/later in the IR cycle to recollide. By contrast, the choice of an XUVphoton energy below the $2 s$ ionization threshold ensures that the $2 s$ electron is born with zero velocity. Thus its recollision trajectories correspond to birthtimes around the penultimate peak of the IR field. As tunnel ionization due only to the IR field is negligible, we assume that only trajectories born during the XUV pulse contribute substantially to the HHG spectrum and- in the shortpulse case- this means only $2 s$ electrons will contribute. This is despite the relatively low probability of ionizing a $2 s$ electron versus a $2 p$ electron.

By contrast, the long pulse does not resolve the contribution of the $2 s$ and $2 p$ electrons. Thus both contributions are present. However, because the ionization probability for a $2 p$ electron is two orders of magnitude larger than for a $2 s$, the HHG yield up to the first cutoff is dominated by $2 p$ electrons, and we only elucidate the $2 s$ contribution above this energy. The initial velocity 


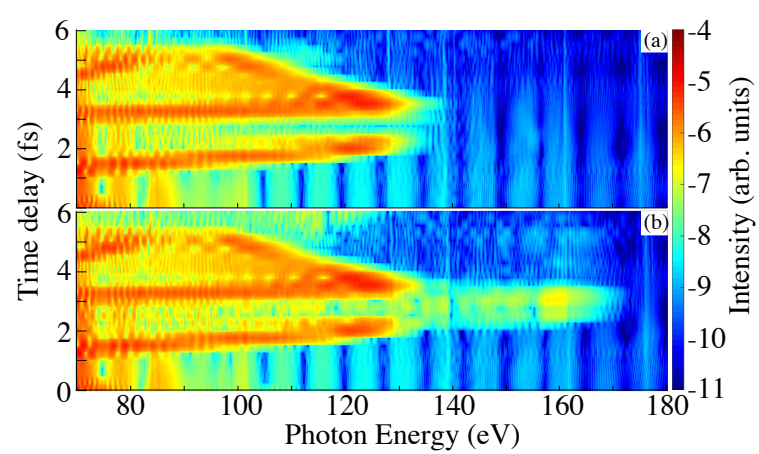

FIG. 4: (Color online) The XIHHG spectrum for $\mathrm{Ne}$ as a function of time delay between the XUV and IR pulse peaks for RMT calculations both (a) neglecting and (b) including the action of a $2 s$ electron.

of the $2 p$ electrons in the field modifies their trajectory from the tunnel-ionization case, reducing the maximum energy of the recolliding electron, and hence the initial $2 p$ cutoff of the HHG spectrum. The maximum energy calculated for the $2 p$ electron is $125 \mathrm{eV}$, in line with the $2 p$ cutoff observed in the spectra (Fig. 2).

In order to confirm the intuition of this simple model we perform calculations for the XUV-photon energies shown in Fig. 1. The yellow squares shown are the predicted cutoff energies for the $2 p$ trajectories as calculated by the model, while orange circle and cross are those calculated for the $2 s$ electron. They are seen to be in good agreement with the RMT results.

To confirm the two-stage ionization mechanism, we refer to Fig. 4, which shows the time-delay scans for two simulations for the 250 as, $45 \mathrm{eV}$ XUV pulse for calculations including or neglecting the action of the $2 s$ electron. The time window in which the $2 p$ recollision is suppressed is clearly seen between 2.5 and 3.5 fs. This corresponds with a drop in overall yield, but an extension of the cutoff energy with the contribution of the $2 s$ electron. The cutoff energy is in line with the prediction of the model calculations shown in Fig. 3. In particular we observe enhancement at the $2 p$ cutoff for time delays around 2 fs and $4 \mathrm{fs}$ which match the predictions of the model (The cutoff energy observed is higher than in the model calculations but within the bandwidth of the XUV pulse, which is not accounted for in the model).

Importantly, the extension of the cutoff due to the $2 \mathrm{~s}$ electron is observed for time delays between 2.25 and $3.5 \mathrm{fs}$, corresponding to the XUV pulse arriving at, or just before, the IR peak. This confirms the proposed two-stage ionization mechanism, wherein the $2 s$ electron is promoted into a $2 s 2 p^{5} n \ell$ Rydberg state before being field ionized at the IR peak. This mechanism warrants further investigation as a scheme may be devised to probe sensitively the lifetimes of excited Rydberg states.

Because the binding energy of inner-valence electrons is not well separated from the ionization potential of the valence shell, XIHHG of inner-valence electrons will not provide substantial gains in cutoff energy. This could be accomplished more easily with the use of higher intensity IR pulses, or indeed with XIHHG of more deeply bound electrons. However, the scheme still represents more promise than may previously have been thought. Due to the relatively small probability of ionizing an inner-valence electron- even with an XUV pulse tuned to the correct binding energy - the outer-valence electron should dominate the HHG process, and indeed, for long XUV pulses we observe that the yield from neon is dominated by the $2 p$ electron contribution up to the $2 p$ cutoff. However, we have shown that with short pulses, whose energy is below the $2 s$ ionization energy, it is possible to selectively suppress the contribution of $2 p$ electrons, and elucidate the contribution of the $2 s$ electron, even if this results in a much lower overall yield.

Thus for attosecond duration XUV pulses delayed appropriately with respect to the IR field, it should be possible to perform HHG spectroscopy of inner-valence electrons. If appropriate pulse profiles, or as-pulse trains can be devised both inner- and outer-valence electrons may contribute on a similar magnitude. This would then facilitate HHG spectroscopy of interference dynamics as previously explored for low energy harmonics [18].

HWH acknowledges financial support from the UK EPSRC under grant no. EP/G055416/1 and the EU Initial Training Network CORINF. This work used the ARCHER UK National Supercomputing Service (http: //www.archer.ac.uk). The data used in this paper may be accessed at Ref. [33].

* Electronic address: andrew. brown@qub.ac.uk; URL:

[1] K. Zhao et al., Opt. Lett. 37, 3891 (2012).

[2] P. M. Paul et al., Science 292, 1689 (2001).

[3] A. D. Shiner et al., Nat. Phys. 7, 464 (2011).

[4] O. Smirnova et al., Nature 460, 972 (2009).

[5] J. Itatani et al., Nature 432, 867 (2004).

[6] P. B. Corkum, Phys. Rev. Lett. 71, 1994 (1993).

[7] E. J. Takahashi et al., Phys. Rev. Lett. 99, 053904 (2007).

[8] M. B. Gaarde et al., Phys. Rev. A 72, 013411 (2005).

[9] F. Brizuela et al., Sci. Rep. 3, 1410 (2013).

[10] O. Smirnova and O. Gessner, Chem. Phys. Lett. 414, 1 (2013).

[11] P. M. Kraus and H. J. Wörner, Chem. Phys. 414, 32 (2013).

[12] J. Biegert et al., J. Mod. Optics. 53, 87 (2006).

[13] G. Gademann et al., N. J. Phys. 13, 033002 (2011).

[14] C. Buth, Eur. Phys. J. D 69, 234 (2015).

[15] C. Buth, M. C. Kohler, J. Ullrich, and C. H. Keitel, Opt. Lett. 36, 3530 (2011).

[16] M. Tudorovskaya and M. Lein, J. Mod. Opt. 61, 845 (2014).

[17] J. Leeuwenburgh et al., Phys. Rev. Lett. 111, 123002 (2013).

[18] A. C. Brown, S. Hutchinson, M. A. Lysaght, and H. W. 
van der Hart, Phys. Rev. Lett. 108, 063006 (2012).

[19] L. Greenman et al., Phys. Rev. A 82, 023406 (2010).

[20] H. Miyagi and L. B. Madsen, Phys. Rev. A 87, 062511 (2013).

[21] L. R. Moore et al., J. Mod. Optics 58, 1132 (2011).

[22] O. Hassouneh, A. C. Brown, and H. W. van der Hart, Phys. Rev. A 90, 043418 (2014).

[23] O. Hassouneh et al., Phys. Rev. A 91, 031404 (2015).

[24] H. W. van der Hart and R. Morgan, Phys. Rev. A 90, 013424 (2014).

[25] T. Ding et al., Opt. Lett. 41, 709 (2016).

[26] K. J. Schafer et al., Phys. Rev. Lett. 92, 023003 (2004).

[27] X. Guan et al., Phys. Rev. A 76, 053411 (2007).
[28] M. A. Lysaght, H. W. van der Hart, and P. G. Burke, Phys. Rev. A 79, 053411 (2009).

[29] L. Torlina and O. Smirnova, Phys. Rev. A 86, 043408 (2012).

[30] L. Torlina, M. Ivanov, Z. B. Walters, and O. Smirnova, Phys. Rev. A 86, 043409 (2012).

[31] P. G. Burke and K. T. Taylor, J. Phys. B. At. Mol. Opt. Phys. 8, 2620 (1975).

[32] J. L. Krause, K. J. Schafer, and K. C. Kulander, Phys. Rev. Lett. 68, 3535 (1992).

[33] A. C. Brown, XUV-initiated HHG in neon, (2016). 10.17034/5a1f395b-d383-44c7-bbf8-5fe31ce35f08, 